Cita bibliográfica: Granell Navarro, J. (2020). Análisis y valoración de la rentabilidad del turismo rural en la provincia de Ávila. Investigaciones Turísticas (19), pp. 121-138. https://doi.org/10.14198/INTURI2020.19.06

\title{
Análisis y valoración de la rentabilidad del turismo rural en la provincia de Ávila
}

\section{Analysis and assessment of the profitability of rural tourism in the province of Ávila}

Javier Granell Navarro iD, Consejería de Educación Comunidad de Madrid, España javigranell@hotmail.com

\section{RESUMEN}

En la última década, el turismo rural ha sido uno de los sectores económicos con mayor desarrollo en la provincia de Ávila. Este trabajo pretende analizar la rentabilidad del modelo de negocio, a partir de una extrapolación de los datos medios de ingresos y costes obtenidos de distintas fuentes, a través de encuestas, estadísticas y declaraciones de los agentes económicos implicados en el sector. La estimación del flujo de caja del proceso mercantil arroja una ratio de rentabilidad muy baja respecto a otro tipo de alquileres más productivos o con menor riesgo. Analizando las partidas, la amortización del inmovilizado es uno de los parámetros determinantes, debido a lo significativo de su porcentaje en los gastos de la actividad. El ajuste del sector, ocasionado por la crisis económica, supuso la salida del mercado del $14 \%$ de los establecimientos de la provincia, y la destrucción del $40 \%$ de los puestos de trabajo del personal empleado, provocando una tensión bajista de los precios. Se exponen las líneas de trabajo futuras y los nichos de mercado que tienen mayor potencial para favorecer el desarrollo del sector, generando mayor productividad en el mismo.

Palabras clave. Turismo Rural; Ávila; Rentabilidad

\section{ABSTRACT}

Rural tourism has been, in the last decade, one of the most developed economic sectors in the province of Avila. This paper aims to analyze the profitability of the business model from an extrapolation of the average data of incomes and costs obtained from different sources, either through surveys, statistics or statements of the economic agents involved in the sector. The estimation of the cash flow of the business process, shows an inferior profitability ratio compared to other types of rents that are more productive or less risky. Analyzing the items, immobilized amortization is one of the determining parameters, due to the significance of its percentage in the activity. The adjustment of the sector caused by the economic crisis has meant the exit of the market of $14 \%$ of the establishments of the province, and the destruction of more than $40 \%$ of the jobs of the employed staff. Triggering downward price pressure. 
Future work lines and market niches that have the greatest potential to favor the development of the sector are presented, generating higher productivity in the sector.

Keywords Rural Tourism; Ávila; Profitability

\section{INTRODUCCIÓN}

El presente trabajo tiene por objetivo analizar los factores que determinan la rentabilidad de los negocios de hospedaje de turismo rural en la provincia de Ávila, valorando qué variables y aspectos de las empresas son más relevantes y cuáles de ellos tienen mayor incidencia en la rentabilidad del proceso comercial.

El turismo rural es un subsector económico muy heterogéneo, por lo que es difícil establecer un modelo estandarizado de tipo de negocio, ya que abarca diferentes tipologías recogidas en el Decreto 75/2013 de 28 de noviembre, tales como: Hotel Rural, Posada y Casa Rural, pudiendo establecerse además diferentes categorías (BOCYL, Boletín Oficial de Castilla y León, 2013). Por otra parte, en la Ley $14 / 2010$ de 9 de diciembre de turismo de Castilla y León, se suprimió la figura más polivalente, que era la de Centro de Turismo Rural (BOE, Boletín Oficial del Estado, 2010) a la que se adscribían establecimientos muy diversos como aquellos compuestos por varias edificaciones con diferentes aforos, $u$ otros que, sin ser estrictamente hoteles rurales, tenían más de 16 plazas de alojamiento.

La normativa que regula la actividad de los establecimientos limita a 50 plazas el aforo máximo de los hoteles rurales y a 16 el de las casas rurales (incluyendo camas supletorias, que nunca podrán suponer más del $50 \%$ de las fijas), siendo por tanto unidades de producción en las que podemos modelizar la instalación tipo para analizar su actuación en el mercado, partiendo de los datos que arrojan diferentes organismos e instituciones presentes en el sector.

\subsection{El turismo rural abulense en la última década}

El desarrollo del turismo rural en Ávila ha estado condicionado por las políticas que, por un lado, se han dirigido a diversificar la dependencia del sector primario (con una agricultura que en muchos casos es de secano y poco productiva), y por otro, a ofertar nuevas fórmulas en el sector terciario con mucho potencial, especialmente en lo referido al turismo rural (Esparcia, 2000), teniendo como target al cliente de Madrid debido a su proximidad.

En las últimas décadas, se ha producido en el conjunto del estado, un crecimiento considerable en la oferta de plazas de alojamiento de turismo rural. Se alcanzó un primer pico en 2012 con 15.389 establecimientos y 142.209 plazas, descendiendo ligeramente en los años sucesivos volviendo a subir posteriormente hasta los 15.669 establecimientos y 147.892 plazas de la actualidad (Hosteltur, 2017). 
La demanda creció también en los últimos años, pero lo hizo de forma más acentuada en los años previos a la crisis económica, alcanzando un primer pico en 2007 con 7.938.623 pernoctaciones, estabilizándose a partir de ese punto y descendiendo en los años posteriores, recuperando e incluso superando dichos valores en 2018 con 11.436 .869 pernoctaciones (Instituto Nacional de Estadística INE, 2019a.). Es significativo que, a pesar del descenso de la demanda desde el año 2007, la oferta continuó creciendo hasta 2012, ya en plena recesión económica.

En la provincia de Ávila la oferta de establecimientos y plazas creció paralelamente al resto de las provincias, pasando de 434 establecimientos y 3.226 plazas en enero de 2006, a 755 y 6.155 respectivamente, en agosto de 2019. Alcanzando los máximos valores de 822 establecimientos y de 6.439 plazas en junio de 2018. La ocupación también sufrió una acusada fluctuación en la última década. El máximo de pernoctaciones y viajeros se produjo en agosto de 2018 con 59.625 y 18.005. Los mínimos se dieron en enero de 2009, con 9.037 pernoctaciones, y en enero de 2010 con 3.825 viajeros (INE, 2019b). Conviene resaltar que los máximos de la demanda y de la oferta durante la crisis estaban separados por cuatro temporadas, habiéndose reducido este descuadre en los últimos años.

Del estudio de la serie de los últimos años, desde principios de 2014 hasta la actualidad, mediante el estadístico "coeficiente de variación" (fig. 1), podemos concluir que las variaciones de su valor en meses consecutivos tienden a reducirse, lo que indica cierta estabilización en el volumen de viajeros y pernoctaciones (INE, 2019c).

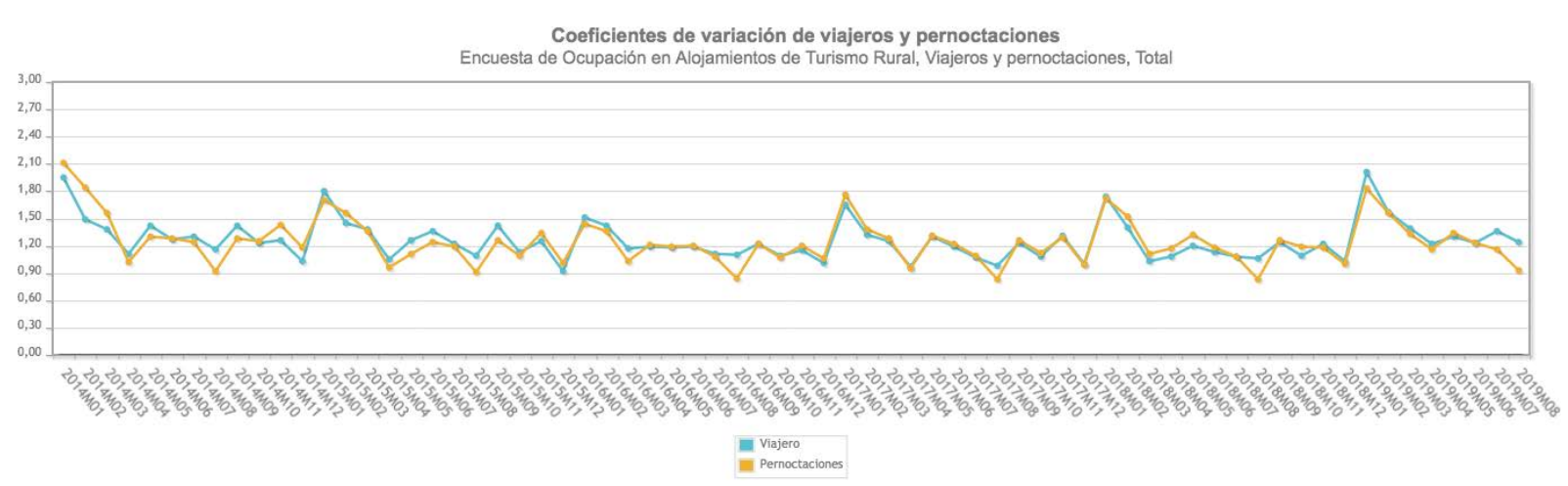

Figura 1. Gráfica de los Coeficientes de Variación viajeros y pernoctaciones Ávila 01/01/2014-31/08/2019. Fuente INE 2019

La evolución del número de personal empleado ha sido también bastante dispar. De los 1.263 trabajadores registrados de enero de 2008, se pasó a los 1.033 en diciembre de 2018. En julio de 2009 se cifró un máximo de 1.696 empleos (fig. 2), pero los reajustes producidos por la crisis económica produjeron una destrucción del $40 \%$ del empleo en el sector (INE, 2019d). 


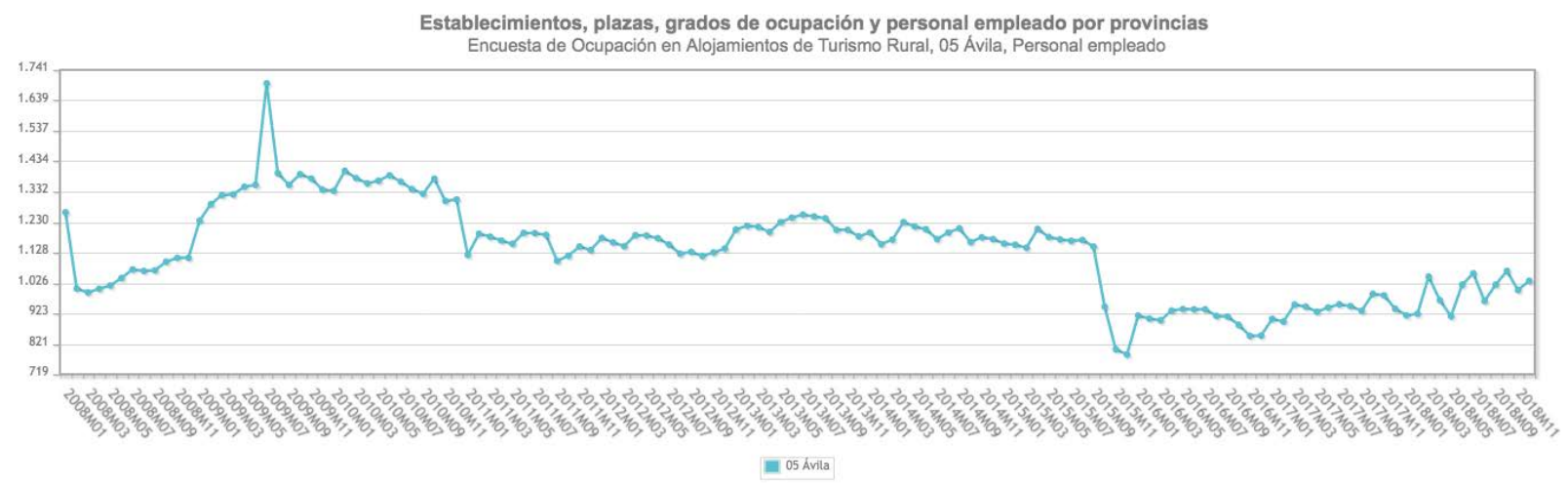

Figura 2. Gráfica del personal empleado en turismo rural en Ávila 01/01/2008-31/12/2018. Fuente INE 2019

Respecto de la demanda, considerando el grado de ocupación por plaza en porcentaje mensual a lo largo de los últimos años, el valor mayor se produjo en agosto de 2006 con el 43,04\% y el mínimo en enero de 2012 con un 5,69\%. El dato de máximo porcentaje de ocupación en el último año del que se tienen datos completos es del 33,57\% en agosto de 2018 (INE, 2019e).

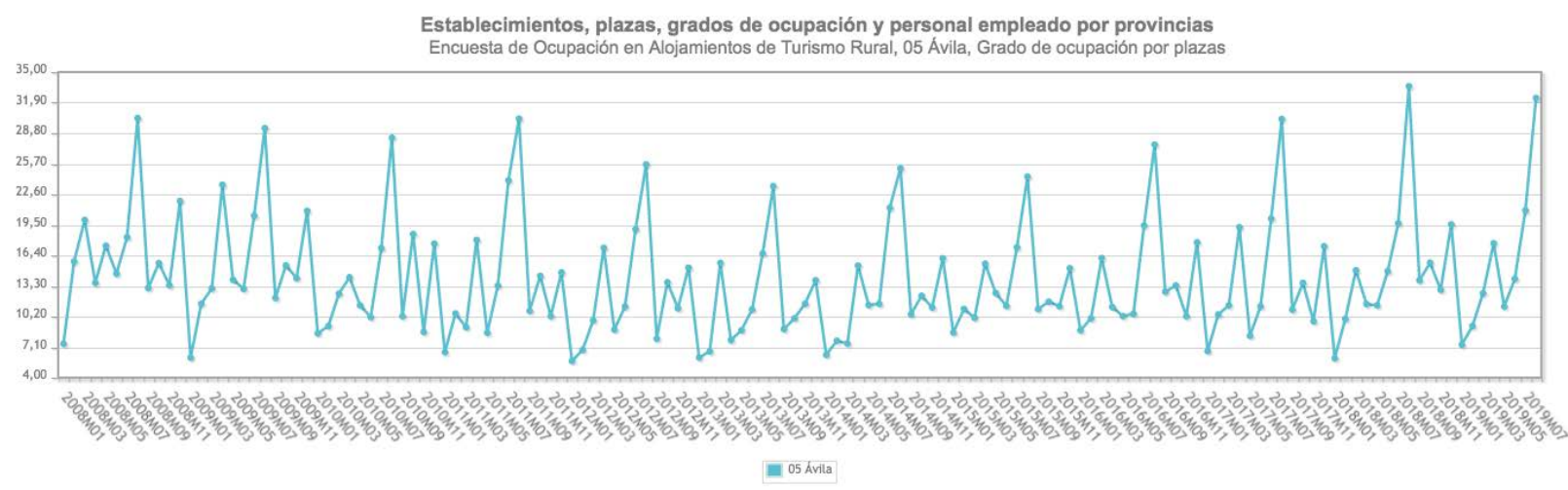

Figura 3. Gráfica del grado de ocupación por plazas en turismo rural en Ávila 01/01/2008-31/07/2019. Fuente INE 2019

Los datos ofrecidos por el INE indican que la demanda creció significativamente al comienzo de 2007 y que esta tendencia continuó hasta 2009. La oferta acompañó a este crecimiento, pero siguió incrementándose hasta 2013, cuando la demanda estaba en franca recesión. De hecho, se puede observar, valorando los datos analizados, que la oferta de establecimientos y plazas casi se duplicó, mientras que los datos respecto de los incrementos de viajeros y pernoctaciones fueron bastante moderados en los primeros años de la crisis económica.

Estos desequilibrios generaron, entre otras consecuencias, una tensión bajista en los precios, que sólo se han recuperado parcialmente en la actualidad. El exceso de oferta es considerado por el $68 \%$ de los propietarios/gerentes de negocios de turismo rural en Castilla y León como el principal problema del sector, estando dicho porcentaje 8 puntos por encima de 
la media nacional. El 10\% de los propietarios han bajado sus precios en la campaña de 2017. (Observatorio del Turismo Rural OTR, 2017a).

\subsection{Entorno del turismo rural en Ávila}

Un aspecto determinante es el de la procedencia del cliente del turismo rural en Ávila. Se cifra en un 54\% (OTR, 2017b) su procedencia desde Madrid, habiendo llegado a alcanzar un $92 \%$ en años anteriores (OTR, 2014a). Esto condiciona un tipo de "escapada" muy ceñida al fin de semana. Todo ello hace que la estancia media, salvo los picos propios de puentes y vacaciones, se encuentre en el entorno de los dos días (fig. 3).

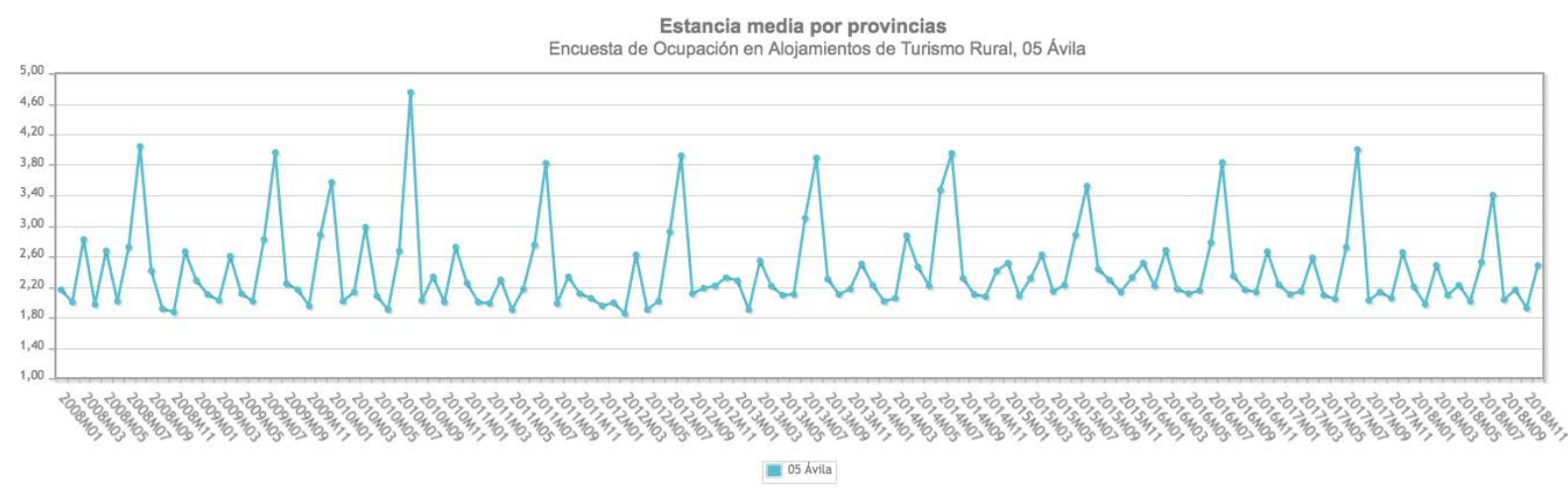

Figura 4. Estancia media en turismo rural en Ávila 01/012008-31/12/2018. Fuente INE 2019

La falta de control de la normativa, el intrusismo y la presencia de establecimientos ilegales es otro problema recurrente para los empresarios del sector, siendo el más importante para el 51,5\% (OTR, 2015a.). Los usuarios perciben una mayor carencia de profesionalidad en los servicios contratados en Castilla y León que en el resto de España (OTR 2017c). En este sentido se han reportado incumplimientos de diferente gravedad de la normativa vigente: desde requisitos para la concesión de diferentes estrellas de categoría, hasta la no adopción de las medidas requeridas para la adecuación a las nuevas tipologías como la de hotel rural. (B.G.R., 2016). La gran extensión de la comunidad, la variedad de administraciones involucradas y la escasez de recursos humanos y materiales, dificulta la supervisión del cumplimiento de la normativa vigente.

De tal manera que, a modo de ejemplo, un Hotel Rural situado en terreno rústico deberá contar con el preceptivo permiso de obras de la Delegación Territorial, informado por el ayuntamiento donde se radique. Así mismo, tendrá que cumplir los requisitos que la Consejería de Turismo dicte en el ámbito de sus competencias, respecto a las características que debe reunir y a la clasificación que se le otorga en función de las mismas. Si además dispone de piscina, ésta se considerará de uso público y deberá seguir las prescripciones del Real Decreto (RD) 742/2013 y del decreto de consejería correspondiente, supervisado por los técnicos de sanidad. Y algo parecido ocurriría con el servicio de restauración.

En caso de ofertar actividades de turismo activo, existe a su vez normativa autonómica que regula su actividad (Decreto 96/2007). Todo este entramado normativo ralentiza y 
entorpece el control y regulación del sector, ya que de su seguimiento, son responsables diferentes servicios, cuerpos y órganos de las administraciones involucradas.

\subsection{Tipología del empresario de turismo rural en Ávila}

El perfil del empresario de turismo rural en Ávila es una mujer en el 55.3\% de los casos, mayor de 47 años, y que compatibiliza en un $72,3 \%$ su dedicación al turismo rural con otro trabajo. La capacidad media de hospedaje es de 13 plazas. La encuesta acerca de las motivaciones del propietario, muestra que la respuesta más veces apuntada de por qué inició este negocio, es: "porque es algo que siempre había querido hacer...", lo que no parece que sea una decisión metódica, planificada y con el análisis previo que todo proyecto empresarial requiere (OTR, 2014b).

Llama la atención el poco grado de asociacionismo que hay en el sector, ya que únicamente el 36\% de los propietarios pertenece a alguna asociación (OTR, 2015b), reconociendo además el 49,2\% no haber pertenecido nunca a ninguna, cifra 4 puntos más alta que en el resto del Estado (OTR, 2017d), lo que indica un cierto aislacionismo en las acciones empresariales adoptadas para mejorar la gestión, promoción, o defensa de intereses comunes del sector.

El Gasto medio en promoción oscila entre 500 y $1000 €$ al año, que se emplean fundamentalmente en el posicionamiento en páginas webs especializadas o centrales de reservas (OTR, 2015c). Esto es debido a que, por las características del sector, es el medio más utilizado para la búsqueda de establecimiento.

La encuesta del Observatorio del Turismo Rural de 2015, indica que, a pesar de la dedicación a tiempo parcial, del intrusismo, de las ilegalidades de muchos establecimientos y de la falta de asociacionismo, los empresarios no perciben como preocupantes aspectos tales como la falta de profesionalismo, o el bajo conocimiento de idiomas y de las tecnologías de la comunicación. Por el contrario, consideran como los mayores problemas del sector: la competencia (por exceso de oferta) y la estacionalidad de la demanda (fig. 5.). Es llamativo que una de las principales «fortalezas» que muchos propietarios creen que tiene su negocio es «el trato que se dispensa a sus clientes», siendo éste, en realidad, un aspecto secundario en la valoración que de las estancias hacen los usuarios, estando por detrás del precio y de la limpieza (OTR, 2014c).

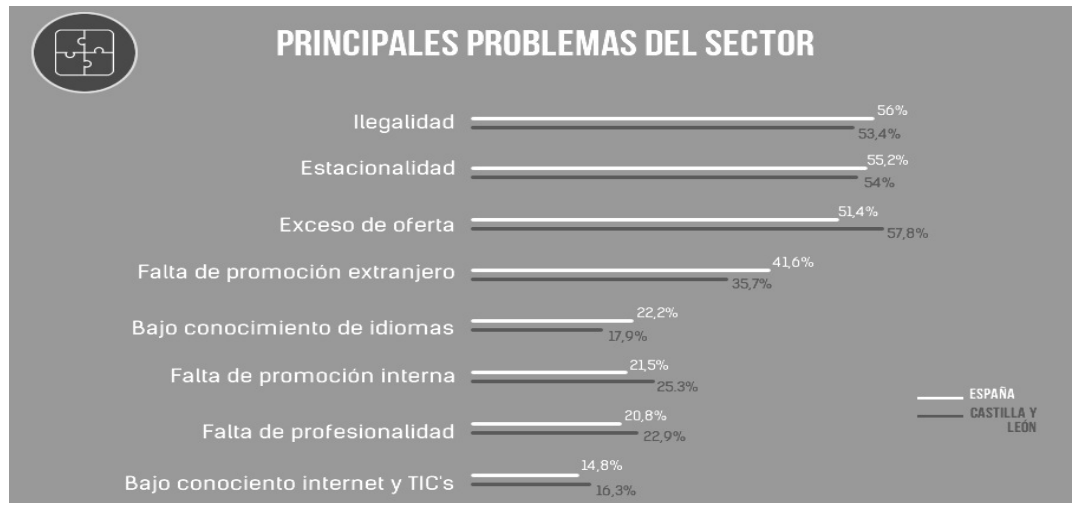

Figura 5. Porcentaje de respuesta, de los propietarios de Castilla y León a la pregunta: ¿Cuál crees que es el principal problema del Turismo Rural? Fuente Observatorio del Turismo Rural. 
El procedimiento de contratación mayoritario $(52,2 \%)$ es el realizado a través de llamadas telefónicas (OTR, 2014d). Sin embargo, cada vez más se recurre a la contratación online, máxime cuando la normativa vigente exige que se transmita fehacientemente determinada información. La dirección de los establecimientos supone mayor dedicación fines de semana y festivos. La promoción, contestación a usuarios y clientes, gestión de reservas y demás funciones administrativas se realiza fundamentalmente on-line.

El nuevo marco legislativo y normativo vigente pretende potenciar el profesionalismo y abordar los problemas de intrusismo del sector, obligando a la presencia permanente de personal en los alojamientos, a la regulación de las titulaciones necesarias para determinados puestos laborales, y al establecimiento de protocolos de contratación, detallando los derechos y obligaciones de usuarios y propietarios. En definitiva, se pretende modernizar el sector para mejorar su eficiencia, incrementando el valor añadido de sus productos y servicios.

\section{EMPRESA-TIPO}

\subsection{Principios generales}

Para analizar la rentabilidad de las empresas estudiadas, vamos a considerar los parámetros que intervienen en los inputs y outputs del proceso productivo. La mayor parte de los inmuebles destinados al turismo rural son propiedad de la persona (física o jurídica) que ejerce la actividad, siendo una minoría los que disponen de inmuebles en condición de arrendatarios. Este hecho condiciona el cálculo de la rentabilidad según consideremos el plazo de amortización de la inversión por la compra del establecimiento, o el gasto por alquiler en su caso.

Es fundamental el cálculo de la inversión en la adquisición del inmueble. Consideramos que el uso exclusivo de índices que no tengan en cuenta el peso de dicha partida, como el EBITDA (Beneficios, Antes de Intereses, Impuestos, Amortizaciones y Depreciaciones) ofrece una información sesgada, al no incluir parámetros como el de las amortizaciones, que afectan muy significativamente en los resultados del ejercicio. Por todo ello, vamos a tomar como referencia el Beneficio Bruto de Explotación (BBE) como indicador para valorar la rentabilidad de las empresas analizadas que es, en muchas ocasiones, muy semejante al Beneficio Antes de Intereses e Impuestos (BAll).

El análisis de la importancia de las actividades y restauración ofertadas en los establecimientos, indica según las fuentes consultadas como la Asociación Nacional de Turismo Activo (ANETA, 2014) y el OTR, que es difícil cuantificar los importes atribuibles a las mismas, ofreciendo datos con un rango inferior a los $20 €$ /día por cliente en los capítulos de actividades y restauración. Del mismo modo, los gastos imputables a dichos servicios son muy difíciles de establecer debido a la heterogeneidad de los mismos, ya que se mueven en un rango muy amplio de costes por compras varias de mercaderías, amortización de equipamientos, salarios de técnicos y especialistas cualificados, etc. 
Los propietarios encuestados manifiestan mayoritariamente, que la oferta de este tipo de servicios es un factor complementario para hacer más atractivo su producto final. Por todo ello no se va a ponderar el flujo de caja de estas actividades en el cálculo final de rentabilidad objeto de este estudio.

\subsection{Ingresos obtenidos}

Las estadísticas que ofrece el estudio, referido a Castilla y León, en el que se analiza el ejercicio de 2017 por parte del Observatorio del Turismo Rural, sobre una encuesta de 15.669 viajeros, con margen de confianza del 95\%, indican que, en Castilla y León el gasto medio por viajero es de $41,2 €$ por día, cifra que permanece prácticamente estable desde hace varios años.

Respecto de la ocupación por plaza en la provincia de Ávila, la media del ejercicio 2018 es del 15,30\% (fig. 6.), lo que supone una ocupación de 55,8 días/año por plaza. Siendo por tanto los ingresos por plaza de 2.298,96 € por año.

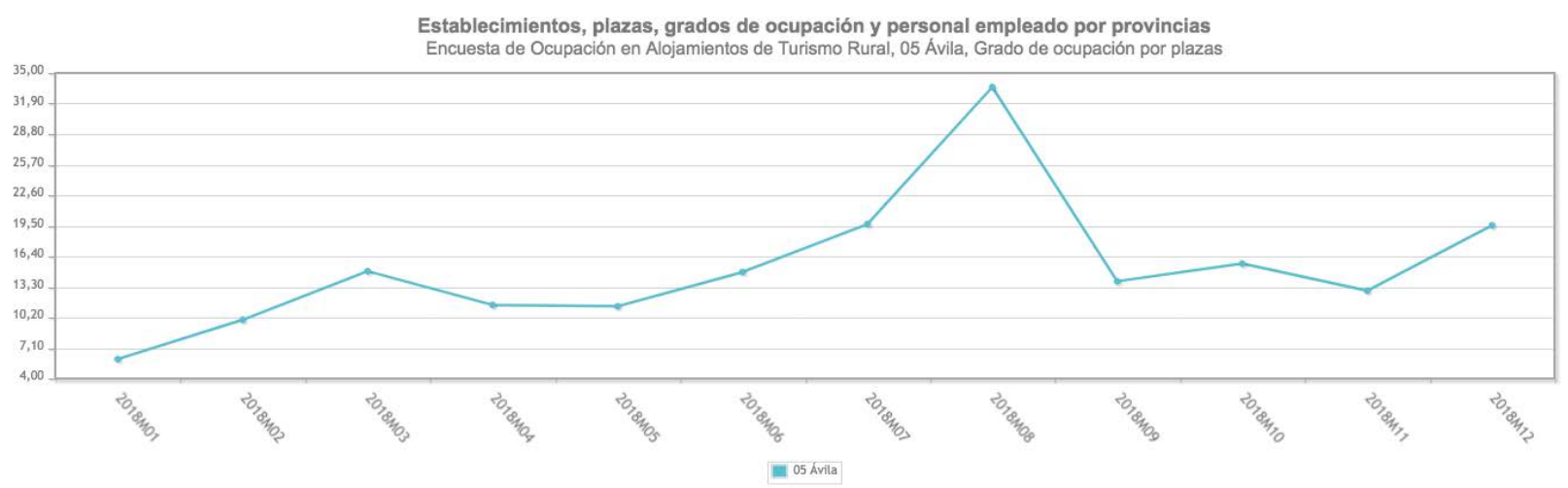

Figura 6. Grado de ocupación por plaza en 2018, provincia de Ávila. Fuente INE 2019

\subsection{Gastos generados}

En el caso que nos ocupa, tomamos como referencia los precios medios por metro cuadrado de vivienda en la provincia de Ávila, ofrecidos por un portal especializado (idealista.es, 2019a). Éstos ascienden a $833 € / \mathrm{m}^{2}$ en el caso de compraventa, y 5,04 $€ / \mathrm{m}^{2}$ mensuales si se trata de alquiler (idealista.es, 2019b). Considerando estas cifras, como referencia orientativa, la amortización del capital principal empleado para la compra del inmueble, requiere aproximadamente 15 años con la cuota del alquiler. Es significativo apuntar que, los precios de los inmuebles han descendido progresivamente en los últimos años, desde un máximo de 1.822 $€ / \mathrm{m}^{2}$ alcanzado en agosto de 2007 , hasta los valores actuales. Del mismo modo, los alquileres han experimentado una rebaja desde un máximo de $6,08 € / \mathrm{m}^{2}$ en junio de 2008 , hasta alcanzar los citados $5,04 € / \mathrm{m}^{2}$. De estas cifras se infiere que el comportamiento del alquiler ha sido más estable y sus oscilaciones menores que los que ha sufrido el mercado de compraventa de inmuebles, ya que la compraventa ha perdido un $42,7 \%$ de su valor, frente al $17,1 \%$ del precio del alquiler. 
Sobre la normativa recogida en el Decreto75/2013, de 28 de noviembre, por el que se regulan los establecimientos de alojamiento de turismo rural en la Comunidad de Castilla y León, tomamos como dato de superficie media por huésped, $20 \mathrm{~m}^{2}$, computando los metros de la habitación-tipo (considerando en el modelo, una habitación doble estándar, que es la más frecuente), así como la parte proporcional de las zonas comunes tales como terrazas, baños y cocina. Estas cifras nos dan un gasto anual de inmueble por plaza de 1.209,60 €, en el caso de alquiler, y de similar magnitud en el caso de compraventa con amortización a 15 años.

Respecto al gasto en personal, calculando la media de plazas ofertadas y trabajadores en el último ejercicio completo, obtenemos la cifra de 6.175 plazas de turismo rural en la provincia de Ávila, que dan ocupación a 994 trabajadores, por lo que podemos estimar una ratio de 6,21 plazas por trabajador. Tomando como referencia el salario recogido en el Convenio Colectivo de Hostelería de Ávila, con validez a partir del 1 de enero de 2018 (Boletín Oficial de la Provincia de Ávila BOPA, 2018), y considerando las variaciones de categoría asimilables a las funciones profesionales que se realizan en un alojamiento de turismo rural (recepción, limpieza, cocina y servicios varios), hemos establecido una retribución tipo de $1200 €$ mensuales brutos, incluyendo la prorrata de pagas extras, es decir $14.400 €$ anuales. Los datos expuestos nos arrojan un gasto anual de personal por plaza ofertada de $2.318,84 €$ en jornada completa. Dado que, tal y cómo se ha mencionado, la mayoría de los trabajadores del sector, lo son a tiempo parcial tomamos como gasto real el 50\% del cálculo a jornada completa, es decir $1.159,42 €$.

Los gastos en los que incurre la empresa-tipo que describimos ascienden en los capítulos de alquiler de inmuebles y personal a 2.369,02 $€$ anuales. Por definición, los gastos fijos no se pueden asociar a un modelo de gasto promedio, pero es una partida importante que conlleva gastos de administración societaria, seguros, mantenimiento de instalaciones, promoción, etc. En este modelo no se incluyen, pero se mencionan porque son otra partida que habría que considerar en un futuro plan de empresa. Otros gastos variables por suministros (agua, gas, electricidad, leña...) ascenderían por plaza a unos 0,80€ por cada día de uso, según el porcentaje de ocupación media del último año. Esto nos da una cifra de 44,64€.

Los gastos cuantificables ascienden a $2.413,66 €$ por plaza y año. Por tanto, el BBE es de $-114,70 €$ / año, según el modelo que se ha establecido a partir del cálculo de los costes por alquiler o adquisición del inmueble (amortización a 15 años), número medio de trabajadores por plaza ofertada y suministros, no valorando gastos fijos imputables al funcionamiento administrativo, ni el flujo de caja de actividades y restauración. Es decir, el resultado de explotación da pérdidas, debido fundamentalmente a las partidas de los costes del inmovilizado y personal.

Para que el resultado arroje beneficios, es necesario que el plazo de amortización del inmueble se prolongue a más de 18 años, según el tipo de interés que se considere. Conviene recordar que las tablas de amortización de la Agencia Estatal Tributaria de viviendas y locales está entre 50 y 100 años (el suelo no se amortiza). 


\begin{tabular}{|l|c|}
\hline Ingresos por alojamiento & $2.298,96 €$ plaza/año \\
\hline Gastos de funcionamiento y personal & $1.403,11 €$ plaza/año \\
\hline Amortización inmueble & $1.204,06 €$ plaza/año \\
\hline Beneficio antes de amortización & $895,85 €$ plaza/año \\
\hline Resultado de explotación & $-114,70 €$ plaza/año \\
\hline
\end{tabular}

Tabla 1. Resumen de ingresos y gastos

\section{DISCUSIÓN}

Para el cálculo del Beneficio Bruto de Explotación, hemos considerado los valores promedio de ingresos y gastos derivados del ejercicio de la actividad mercantil, y hemos tomado como referencia los precios de alquiler (o en su caso, compraventa) del $\mathrm{m}^{2}$ en la provincia objeto del estudio. Este modelo de cálculo tiene una desviación según la zona que se trate, ya que el precio del $\mathrm{m}^{2}$ oscila entre los poco más de $400 €$ en pueblos pequeños, y los más de $1.100 €$ en la capital de provincia (Idealista.es, 2019a ídem). Lo mismo ocurre con la cuantía del alquiler, figura que es muy excepcional en el sector que nos ocupa, y que se da mayoritariamente cuando se trata de un alquiler con opción a compra. En ambos casos, al incluir los valores de toda la provincia, el dato calculado puede mayorar el valor real. Con lo que es posible que los gastos derivados de su adquisición o arriendo sean algo más reducidos que los tomados en la modelización del análisis.

Dado que se trata de un mercado relativamente reducido, el valor de un inmueble que se destina a una actividad de hostelería se establece, en gran medida, en función del beneficio de explotación del mismo u otra ratio indicativa de su rentabilidad. De esta manera quizá sería pertinente hacer la valoración del precio de mercado del inmueble partiendo del valor de la cuenta de resultados del negocio que opera en él, incrementándose o aminorándose en función de lo rentable que éste sea.

Los datos de gasto por viajero y día que hemos tomado como base de cálculo parten de las entrevistas que el Observatorio del Turismo Rural hace sobre los clientes y usuarios de los servicios analizados. Consideramos más fiable este dato que el Precio de Venta al Público (PVP) de los establecimientos que recoge el INE, ya que estos valores son siempre los máximos, y hemos observado que en el sector se ofertan constantemente promociones y rebajas sobre los citados PVP (toprural, 2016). Así mismo, el 30\% de los propietarios manifiesta tener una política de precios variable y negociable con los clientes (OTR, 2107e).

Otro aspecto que en el modelo es esencial para el cálculo de los ingresos, es el de la ocupación media por plaza. Dado que este dato se remite al INE a través de una encuesta de obligado cumplimiento por los responsables de los establecimientos, su valor real suele ajustarse al alza, ya que hay un porcentaje de hospedajes no declarados por diversos motivos. El cotejo de los datos aportados con el de la ocupación real de las plazas es prácticamente inexistente, lo que propicia que se den desajustes, sobre todo en establecimientos pequeños o en aquellos con poco personal. Este factor se acrecienta debido a que la duración de la ocupación de plazas en la provincia estudiada está muy vinculada al fin de semana, existiendo por consiguiente una alta rotación de usuarios. Por todo ello es factible que la ocupación sea mayor 
que la utilizada para el cálculo de la rentabilidad según el modelo que hemos establecido, existiendo en todo caso un amplio rango en la variabilidad de ocupación según el alojamiento de que se trate.

Tal y como se ha expuesto, los ingresos debidos a restauración y actividades de turismo activo son muy variables. Existen establecimientos especializados en diferentes servicios que hace que el porcentaje de ingresos debidos a los mismos sea significativo en el volumen de facturación total. Un análisis en dicho aspecto pasaría por caracterizar las diferentes tipologías de establecimiento en función de los servicios que presta. Es frecuente que la oferta de este tipo de actividades se realice, y su servicio se preste por una empresa especializada, permitiendo al establecimiento de turismo rural comisionar el servicio u ofrecerlo como un complemento atractivo dentro de su propia oferta, incrementando así el valor añadido de la misma.

Los gastos variables atribuibles a suministros están ajustados, y su variabilidad estará en función de las calidades constructivas y de mantenimiento de los establecimientos. Los gastos fijos derivados del funcionamiento administrativo de la actividad mercantil son también fácilmente cuantificables y no suponen, en definitiva, una partida de especial trascendencia a la hora de valorar la rentabilidad de la empresa.

\section{1. ¿Son rentables las empresas de turismo rural en la provincia de Ávila?}

Esta pregunta es la esencia de éste análisis y para ser contestada adecuadamente debe tener en cuenta una serie de premisas:

La rentabilidad esperada en una actividad mercantil depende de varios factores, tanto intrínsecos (riesgo asumido, dificultad técnica, necesidades específicas de la actividad, etc.) como extrínsecos (estabilidad institucional, entorno micro y macroeconómico, etc.). Considerando el sector de hostelería y turismo la rentabilidad media de la inversión, según el BBE, oscila en un rango del $8 \%$ al 22\% (Pérez \& Simarro, 2008). Sobre estos porcentajes es factible estimar indicadores como el Valor Actual Neto (VAN), Tasa Interna de Retorno (TIR), o el Retorno de la Inversión (conocido por su acrónimo inglés ROI). En este sentido, y siguiendo los estudios elaborados por el OTR, es llamativo que solamente los empresarios de turismo rural de la Comunidad Valenciana manifiestan mayoritariamente que miden el ROI como referencia de la rentabilidad de su inversión. Este espectro relativamente amplio de rentabilidad, al que hemos hecho referencia, se debe a la heterogeneidad de un sector condicionado por una gran competencia, la poca cualificación general del personal empleado, y el tamaño de la empresa (Acedo et al., 2005)

Dado que la mayoría de los propietarios del sector compatibiliza esta actividad profesional con otra, las ratios de rentabilidad se distorsionan, valorando que obtener ingresos supone de por sí ser rentable, sin analizar el proceso comercial.

Muchas empresas del sector no contabilizan el gasto por la inversión en inmovilizado en su valor real, sino que lo minimizan u obvian. Al tratarse de inmuebles sin cargas, o con cuotas de poca cuantía, no cifran correctamente la amortización. De esta manera el BBE puede resultar engañoso al estar distorsionado por no incluir una partida tan significativa. De hecho, 
al preguntar al empresario por los motivos que le llevaron a desarrollar un proyecto empresarial en el sector, los datos que arroja el Observatorio del Turismo Rural indican que, en un porcentaje significativo, según cada Comunidad Autónoma, lo hicieron por recibir una casa de pueblo en herencia.

Los datos de ocupación que arroja el INE son, como se ha expuesto, tendentes a disminuir la ocupación real, lo que unido al elevado número de establecimientos ilegales, hace que la ocupación efectiva sea posiblemente mayor.

Partiendo de las premisas anteriores podemos valorar la rentabilidad de las empresas en función de los factores que intervienen en el proceso comercial. Considerando los ingresos, éstos dependerán de la rotación de usuarios y precios establecidos, y de los servicios añadidos de hostelería y actividades varias. Atendiendo a los gastos, el principal fijo es el valor atribuido al inmueble y la amortización que se establezca del mismo. La cuenta de resultados dará un valor de beneficios en función de la inversión realizada, por lo que será decisión de la dirección de la empresa asumir la tasa de rentabilidad que haga operativo el modelo de negocio. Baste como referencia indicar que, en Ávila, negocios considerados de riesgo reducido, tales como el alquiler de viviendas o locales, tienen una rentabilidad del $5,5 \%$ y $8,1 \%$ respectivamente (idealista news, 2017).

Respecto a otro tipo de negocios muy extendido en el ámbito de la hostelería, como es el de la participación a modo de franquiciado en una red de establecimientos, la rentabilidad obtenida por la inversión varía entre un $7 \%$ y un $19 \%$ según el coste de oportunidad del capital que intervenga en cada modelo (Roldán et al., 2010). Estos datos indican que la valoración de una inversión dependerá del beneficio esperado, y en este sentido, por término medio, las ratios que el turismo rural ofrece en la provincia de Ávila no son competitivas si consideramos todos los factores que intervienen en su desarrollo. El cálculo de los BBE o del ROI, obviando el coste real del inmueble y atribuyendo un valor subjetivo a partidas intangibles difícilmente mesurables (fondo de comercio, know how, fidelización de clientes...), supone una distorsión de la rentabilidad efectiva de la empresa. Otra variable que hemos de considerar es el apalancamiento financiero que una inversión de este tipo suele producir en una pequeña empresa, lo que resta flexibilidad o capacidad de adaptación ante escenarios cambiantes.

Por todo ello, partiendo del modelo analizado según los datos aportados por el INE y triangulados con los ofrecidos por otras instituciones del sector, la rentabilidad del turismo rural en la provincia de Ávila, en términos medios, no ofrece unos resultados que inversiones con coste de oportunidad incluso menores, están produciendo en la actualidad.

\section{FUTURAS LÍNEAS DE TRABAJO}

La reciente revisión del marco legislativo que regula el turismo rural en Castilla y León supone un intento de mejora de calidad del sector. La atención puesta en la formación del personal y el control e inspección efectiva del cumplimiento de la normativa, son acciones importantes para la restructuración del sector. Las estrategias empresariales deben dirigirse a la mejora de los índices de productividad, lo que pasa por profesionalizar la gestión para mejorar la eficiencia de los procesos mercantiles (Lillo et al, 2007). 
Se deben estudiar las tendencias y los gustos del cliente con el fin de potenciar y desarrollar aquellas líneas de trabajo más productivas. Actualmente el precio sigue siendo el aspecto más influyente al hacer la reserva, seguido de las fotos y opiniones (fig. 7). Factores, estos dos últimos, muy volátiles, por lo que hay margen de actuación en los planes de marketing, en la mejora de la oferta, promoción, y en la venta de productos y servicios.

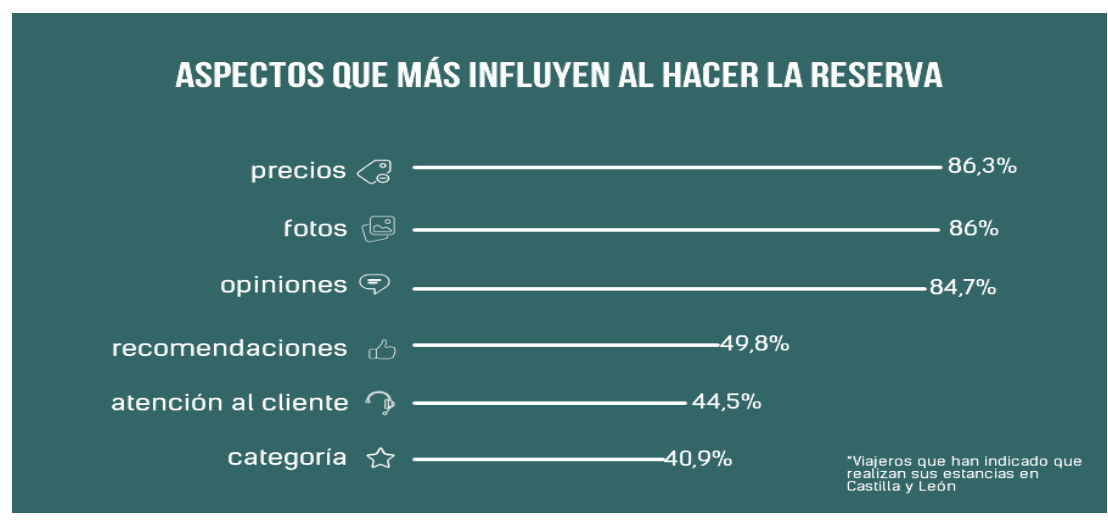

Figura 7. Pregunta a viajeros ¿qué aspectos influyen más a la hora de la reserva en un alojamiento de turismo rural. Fuente Observatorio del Turismo Rural. 2015

\subsection{Estrategias de actuación}

\subsubsection{Turista Extranjero}

Un cliente potencial fundamental para favorecer el crecimiento de la productividad, y por tanto el de ingresos, debe ser el turista extranjero, que gasta más de 3 veces la cantidad diaria de un turista nacional, y que además prolonga significativamente la duración de sus estancias (Ortega y Rodríguez 2005). En este sentido podemos observar la evolución del turismo rural de usuarios extranjeros en Ávila en lo que va de año, existiendo un pico claro en verano (fig. 8). Es evidente que existe un amplio margen de mejora que pasa por potenciar las «oportunidades» y «elementos diferenciales» que caracterizan el entorno del sector.

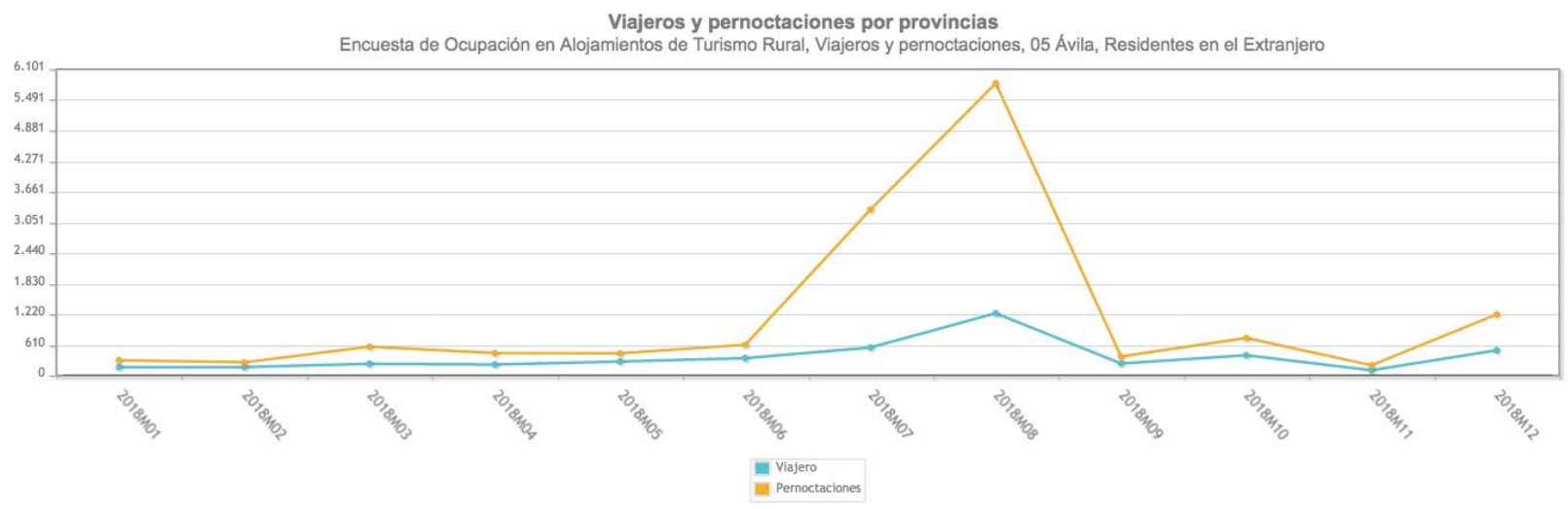

Figura 8. Evolución de viajeros y pernoctaciones extranjeros en la provincia de Ávila en 2018. Fuente INE. 2019

La captación del turista extranjero es una oportunidad que requiere, además de la promoción e información de la oferta en origen, la estandarización de procedimientos que 
adecúen la calidad del servicio (real y percibida) respecto de los parámetros que manejan los usuarios "target» a los que destina. Es decir, se ha de avanzar en el implemento y adopción de programas ISO (Organización Internacional para la Estandarización) que certifiquen la calidad. Estas actuaciones pasan, implícitamente, por profesionalizar la gestión.

\subsubsection{Turismo cultural y gastronómico}

Es un nicho tradicionalmente trabajado, pero la falta de previsión, experiencia, profesional y capacidad de adaptación respecto de la demanda, hace que muchos alojamientos subcontraten estos servicios, o bien, aquellos que lo ofrecen, no lo hagan con suficiente calidad. Los datos que arroja el Observatorio del Turismo Rural, indican que el 17,6\% del turista elige su escapada por "vivir experiencias eno-gastronómicas». Existe una amplia red de establecimientos de restauración en la provincia que, sin embargo, mantienen una política de marketing poco coordinada con el de la oferta de hospedaje. Mejoras en las campañas de promoción conjuntas (restauración y hospedaje) crearían, sin duda, sinergias favorables para el sector, en términos globales.

El turismo cultural es otro nicho que tradicionalmente ha abanderado Castilla y León. En el caso de Ávila, hay tres ciudades Patrimonio de la Humanidad en un radio de $100 \mathrm{Km}$. La riqueza cultural de la provincia es una clara fortaleza a tener en cuenta en cualquier estrategia de actuación. El análisis de la oferta en los establecimientos pone de manifiesto la descoordinación que existe en la respuesta a la demanda del mercado, máxime en lo que atañe a paquetes cerrados adaptados a la disponibilidad temporal y a los gustos del cliente potencial.

\subsubsection{Turismo activo}

El nicho del turismo activo o deportivo es una apuesta muy clara en comunidades como Aragón (Lacosta, 2004), donde se ha trabajado tradicionalmente (inicialmente como estrategia para complementar la oferta invernal y atenuar la estacionalidad de la misma), convirtiéndose en una seña de identidad del turismo rural de la región. En Castilla y León, comparativamente, se trata de un subsector que no está desarrollado en todo su potencial, siguiendo una política errática en la oferta de actividades que, una vez más, adolecen de profesionalidad en su ejecución práctica (entendida como la planificación, utilización de equipamiento, formación y dinamización de la misma).

Estos factores han propiciado que la oferta de actividades sea muy cambiante. Así, hace unos años era muy frecuente la oferta de actividades como paint-ball o rutas en quads, que son hoy en día mucho más escasas. Del mismo modo ocurre con actividades de «bajo coste» como el tiro con arco o las carreras de orientación, que se ofrecen por no suponer una inversión importante para el establecimiento, pero su práctica no conlleva necesariamente un incremento del valor añadido del servicio prestado.

Otras actividades frecuentes en los catálogos son las rutas a caballo y la escalada. Ambas requieren una inversión considerable y por tanto un plan de negocio adecuado que diseñe las actuaciones a desarrollar, así como el cronograma de las mismas. Este tipo de actividades, 
una vez están consolidadas, son las que aportan mayor valor añadido y productividad. Otro segmento de actividades como las náuticas, las aéreas o las de montañismo dependerán de las posibilidades del entorno.

Un aspecto a tener en cuenta es la incompatibilidad de determinadas actividades. Por ejemplo, ofertar paint-ball, y observación de aves, en el mismo emplazamiento, resulta contraproducente, tanto por los perfiles de los clientes como por los entornos a los que afecta la realización de las mismas. El medio natural de la provincia de Ávila ofrece una importante oportunidad de práctica de ocio activo, lo que supone una línea de trabajo a tener en cuenta. La práctica de este tipo de actividades implica el control y el cumplimiento del marco normativo, algo que reduciría el intrusismo y la ilegalidad de muchos ofertantes (García, 2010).

\subsubsection{Turismo de reuniones}

Este nicho abarca el turismo de empresa, convenciones y eventos. Este tipo de servicios requiere disponer de instalaciones suficientes para su realización, lo que supone una inversión importante y, consecuentemente, una adecuación respecto de la visión a medio y largo plazo de la empresa.

Se trata de un nicho de mercado copado por los hoteles tradicionales, que disponen de una ventaja competitiva en lo que a instalaciones se refiere. Los servicios destinados a eventos (bodas, reuniones familiares, etc.) de carácter menos formal y convencional, tienen una presencia significativa en relación a la oferta tradicional en el sector del turismo rural. El reto de desarrollo de este nicho pasa por entrar en los canales de promoción y distribución de los servicios, donde los hoteles tienen una presencia muy consolidada.

\section{CONCLUSIONES}

El turismo rural en la provincia de Ávila, al igual que en el resto de la región, pasa por una etapa de readaptación a un nuevo escenario de mercado. De los datos analizados podemos extraer las siguientes conclusiones:

1. La oferta de establecimientos creció significativamente a un ritmo superior a lo que lo hizo la demanda de hospedajes durante los últimos 10 años. La crisis económica ha propiciado la salida del mercado de un $14 \%$ de establecimientos, y de más de un $40 \%$ del personal empleado, habiéndose recuperado solamente la oferta de plazas.

2. El ajuste del sector se ha hecho fundamentalmente por reducción de personal empleado y bajada de precios, es decir disminuyendo costes sin incrementar ingresos.

3. La rentabilidad de las empresas de turismo rural está, porcentualmente, muy condicionada por el tipo de amortización de los inmuebles donde se desarrolla, que en la mayoría de los casos son de titularidad del ejercitante de la actividad mercantil. Tomando como referencia de precios de los inmuebles los valores medios de la provincia, es necesario un plazo de amortización superior a 15 años para obtener beneficios, y del doble para que la tasa de rentabilidad sea algo más competitiva. 
4. La evolución del sector se está encaminando a una consolidación de aquellas empresas más productivas y profesionalizadas en su gestión. La reciente legislación vigente establece categorías de hospedajes en función de la calidad de los mismos, al tiempo que concreta las obligaciones de los ejercitantes de la actividad y especifica los derechos y deberes de los clientes. Todo ello tendente a la mejora de la calidad y a la erradicación de los establecimientos que incumplan la normativa o presenten algún tipo de ilegalidad.

5. Existen diferentes nichos de mercado infra-explotados que tienen un gran potencial en el desarrollo futuro del sector, requiriendo para su desarrollo un plan a medio plazo, de formación, promoción e inversión.

\section{REFERENCIAS}

Ayala J.C., Rodriguez, J.E., Acedo M.A. (2005) Rentabilidades, endeudamiento y coste de la deuda de las pymes, análisis empírico de las empresas por tamaños. Revista Europea de Dirección y Economía de la Empresa 14, (4) pp. 185-200.

ANETA (2014) Informe sobre el turismo activo en España. Recuperado de: http://www.aneta. es/informe-turismo-activo-2014/

B.G.R. 22 de abril de 2016. Diario de Burgos. Abren 3 expedientes en las primeras inspecciones a casas rurales con estrellas Recuperado de: http://www.diariodeburgos.es/ noticia/Z4DFA151B-ADF4-3E8B-573F29ABE29752B5/20160422/abren/3/expedientes/ primeras/inspecciones/casas/rurales/estrellas.

BOE no 317 de 30 de diciembre de 2010; p.108894.

BOPA no 203 de 18 de octubre de 2013; p.78636.

Chicharro E., Galve A. (2009) Alojamientos rurales en España: entre el crecimiento acelerado y el peligro de una sobredimensión. Serie geográfica, 15; pp. 125-137.

Decreto 75/2013 de 28 de noviembre por el que se regulan los establecimientos de alojamiento de turismo rural en la Comunidad de Castilla y León.

Decreto 96/2007, de 27 de septiembre, por el que se regula la ordenación de las empresas de turismo activo de la Comunidad de Castilla y León.

Esparcia J. (2000) The LEADER Programme and the Rise of Rural Development in Spain. Sociologia Ruralis, 40 (2).

García P.J. (2010) Turismo activo y medio ambiente: Una implicación necesaria. Aspectos jurídicos. Cuadernos de Turismo, 26; pp. 153-176.

Hosteltur (2017) Radiografía del turismo rural en España, ¿un sector rentable? Recuperado de: https://www.hosteltur.com/121363 radiografia-turismo-rural-espana-sector-rentable.html.

idealista (2019a) Precio medio viviendas en venta provincia de Ávila. Recuperado de: https:// www.idealista.com/venta-viviendas/avila-provincia/.

idealista (2019b) Precio medio viviendas en alquiler provincia de Ávila. Recuperado de: https:// www.idealista.com/alquiler-viviendas/avila-provincia/.

Idealista news (2017) Rentabilidad del alquiler de viviendas y locales por provincias. Recuperado de: https://www.idealista.com/news/inmobiliario/vivienda/2017/ 
10/20/748540-la-rentabilidad-de-la-inversion-de-vivienda-se-situa-en-el-7-1-en-eltercer

INE (2019a) Encuesta de ocupación en establecimientos de turismo rural, evolución de la oferta total nacional 2006-2018.

INE (2019b) Encuesta de ocupación en establecimientos de turismo rural. Número de establecimientos y plazas en la provincia de Ávila 2014-2019.

INE (2019c) Coeficientes de Variación viajeros y pernoctaciones provincia de Ávila 2014-2019. INE (2019d) Encuesta de ocupación en establecimientos de turismo rural. Personal empleado, provincia de Ávila 2008-2018.

INE (2019e) Encuesta de ocupación en establecimientos de turismo rural, porcentaje de ocupación por plaza, provincia de Ávila 2008-2019.

Lacosta A.J. (2004) La configuración de nuevos destinos turísticos de interior en España a partir del turismo activo y de aventura (1992-2001). Cuadernos Geográficos, 34: pp. 11-31.

Lillo A., Ramón A.B., Jimenez M. (2007) El capital humano como factor estratégico para la competitividad del sector turístico. Cuadernos de Turismo, 19; pp. 47-69.

Molina A., Martín-Consuegra D., Esteban A., Díaz E. (2007) Segmentación de la demanda turística: un análisis aplicado a un destino de turismo cultural. Revista de Análisis Turístico, 4; pp. 36-48.

OTR (2017a) «Grupo de investigación sobre viajeros y alojamientos rurales». Universidad de Barcelona; p. 18.

OTR (2017b) «Grupo de investigación sobre viajeros y alojamientos rurales». Universidad de Barcelona; p. 2

OTR (2017c) «Grupo de investigación sobre viajeros y alojamientos rurales». Universidad de Barcelona; p. 21

OTR (2017d) «Grupo de investigación sobre viajeros y alojamientos rurales». Universidad de Barcelona; p. 17

OTR (2017e) «Grupo de investigación sobre viajeros y alojamientos rurales». Universidad de Barcelona; p. 2

OTR (2017f) «Grupo de investigación sobre viajeros y alojamientos rurales». Universidad de Barcelona; p. 22

OTR (2014a) «Grupo de investigación sobre viajeros y alojamientos rurales». Universidad de Barcelona; p. 267.

OTR (2014b) «Grupo de investigación sobre viajeros y alojamientos rurales». Universidad de Barcelona; p. 205.

OTR (2014c) «Grupo de investigación sobre viajeros y alojamientos rurales». Universidad de Barcelona; p. 242.

OTR (2014d) «Grupo de investigación sobre viajeros y alojamientos rurales». Universidad de Barcelona; p. 265.

OTR (2015a) Informe Castilla y León», Universidad de Barcelona; p. 8.

OTR (2015b) «Informe Castilla y León», Universidad de Barcelona; p. 11.

OTR (2015c) «Informe Castilla y León», Universidad de Barcelona; p. 10.

Ortega E., Rodriguez B. (2005) Importancia de la comunicación en los destinos turísticos internaciones: la percepción de los turistas extranjeros en los destinos españoles. Cuadernos de Turismo, no 15; pp. 169-188. 
Pérez A.S., Slimarro R. (2008) Análisis comparativo de la rentabilidad y productividad de las empresas hoteleras en el mercado europeo. Revista de análisis turístico, 5; pp. 4-15.

$\mathrm{RD} 742 / 2013$, de 27 de septiembre por el que se establecen los criterios técnico-sanitarios de las piscinas.

Roldán F.J., Navarro A., Díez de Castro E.C., Rodríguez C., Guisado M. (2010) Estudio del Performance de los Grupos Estratégicos del Sistema de Franquicia español. Investigaciones Europeas de Dirección y Economía de la Empresa, 16, № 2; pp. 43-62.

Toprural (2016) Ofertas de casas rurales. Recuperado de: http://www.toprural.com/info/ofertas-casas-rurales I.html 